NASA Technical Memorandum 104523

\title{
Modern CFD Applications for the Design of a Reacting Shear Layer Facility
}

S.T. Yu

Sverdrup Technology, Inc.

Lewis Research Center Group

Brook Park, Ohio

and

C.T. Chang and C.J. Marek

National Aeronautics and Space Administration

Lewis Research Center

Cleveland, Ohio

Prepared for the

29th Aerospace Sciences Meeting

sponsored by the American Institute of Aeronautics and Astronautics

Reno, Nevada, January 7-10, 1991 


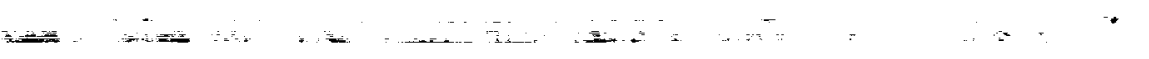




\title{
Modern CFD Applications for the Design of a Reacting Shear Layer Facility
}

\author{
S.T. Yu* \\ Sverdrup Technology. Inc. \\ Lewis Research Center Group \\ Brook Park, Ohio 44142 \\ C.T. Chang $†$ and C.J. Marek $\ddagger$ \\ National Aeronautics and Space Administration \\ Lewis Research Center \\ Cleveland, Ohio 44135
}

\begin{abstract}
The RPLUS2D code, capable of calculating high speed reacting flows, has been adopted to design a compressible shear layer facility. In order to create reacting shear layers at high convective Mach numbers, hot air streams at supersonic speeds, render by convergingdiverging nozzles, must be provided. A finite rate chemistry model is used to simulate the nozzle flows. Results are compared with one-dimensional, chemically equilibrium solutions. Additionally, a two equation turbulence model with compressibility effects has been successfully incorporated with the RPLUS code. The model has been applied to simulate a supersonic shear layer. Preliminary results show favorable comparisons with the experimental data.
\end{abstract}

\section{INTRODUCTION}

Recent national interest of trans-atmospheric vehicle has rekindled the hypersonic research. For this vehicle, a supersonic combustion ramjet (scramjet) engine was proposed to provide the power. Inside this scramjet engine, compressible mixing layers with chemical reactions are important phenomena. The performance of the engine will depend on the supersonic mixing and the flame holding of shear layers.

The behavior of incompressible mixing layers has been studied extensively. However, additional study is required to understand the compressibility and chemical reaction effects at the high speeds. Recognizing the importance of the supersonic reacting shear layer, a test rig is being designed and built at the NASA Lewis center to study the flow physics. This current paper reports the design process of the test rig using modern

* Research Engineer, Member AIAA.

$\dagger$ Aerospace Engineer, Member AIAA.

$\ddagger$ Senior Research Scientist.
CFD methods and the development of the $k-\epsilon$ model with compressibility effects for the simulation of high speed shear layers.

The experiment is setup to study the mixing of ox. idizer and fuel streams in reacting shear layers for various density, velocity ratios, and Mach number. The primary goal is to understand the effects of the compressibility upon mixing and combustion in a fundamental way. Therefore, a two-dimensional shear layer facility is designed for its simplicity to quantify the flow physics. As shown in Fig. 1, the envisioned facility consists of a two-stream wind tunnel with two independent gas supplies. After passing through flow-management devices located upstream, each gas stream expands to its predetermined Mach number by means of a contoured center body and tunnel walls. Various combinations of flow conditions of high-speed stream and low-speed stream allows for the systematic study of mixing and reactions of compressible shear layers.

Currently, there are several test facilities in the U.S. to study compressible reacting shear layers. Mungal et al. ${ }^{1}$ designed and built a relatively large facility at Stanford University. The test section is $10 \times 5.5 \times 48 \mathrm{~cm}$. Vitiated air at supersonic speed is used as high-speed (oxidizer) stream while hydrogen mixed with inert gas is used as the low-speed (fuel) stream. The facility is a blow down type with typical a run time of 30 seconds.

Dimotakis et al. ${ }^{2}$ at Cal. Tech. built a blowdown type facility in which premixed volume of hydrogen in nitrogen and fluorine in nitrogen are discharged through sonic orifices, maintaining constant mass flow rate in each of the free streams. Then both streams expand to predetermined Mach numbers and meet at the tip of splitter plate. The chemical reaction utilized in their experiment is the fastest one known and it reacts at room temperature. Therefore, no preheating system is needed. Fast chemistry eliminates the issue of the finite rate chemistry. However, fluorine is highly corrosive; tedious preparation and cleanup processes limit the availability of the facility.

Dutton et al..$^{3}$ at University of Illinois designed and 
built a blow-down facility. A commercially available vitiation furnace fueled by natural gas is used. The furnace is operated in a fuel rich condition to elevate the total temperature of the hot stream. The combustion process of methane and air in fuel rich condition produces large amounts of hydrogen which is then used as fuel for the reacting shear layer. By adopting this method, one bypasses the difficult safety procedure for handling hydrogen. However, the chemistry is complex and can not be easily controlled.

In our study, the National Aerospace Plane (NASP) type application is simulated. In order to obtain detailed measurement of the whole flow field, the facility is envisioned to be a continuous wind tunnel with a typical run time of 30 minutes to 1 hour. The shear layer consists of a high-speed, supersonic, vitiated air (oxidizer) stream merging with a low-speed hydrogen (fuel) stream mixed with inert gas (nitrogen) at sonic or subsonic speed. For the oxidizer stream, compressed air at total pressure about $30 \mathrm{~atm}$ is continuously provided by a compressor. The total temperature of this high-speed stream is elevated to ensure the ignition of the shear layer. As illustrated in the Fig. 2, the heating process of this stream is composed of two parts, namely, a heat exchanger and a vitiation facility. The high pressure air first flows through the heat exchanger and is heated up to about $860 \mathrm{~K}$, and additional heat is needed to further raise its total temperature. A vitiation heating system in which hydrogen and oxygen are burned at stoichiometric ratio introduces hot combustion products into the air stream and raises its temperature. In doing so, the chemistry of the vitiation process is consistent with that of the shear layer test and no additional complexity of chemical kinetics is introduced into the system.

Finally, the hot air stream flows through a converging diverging nozzle to reach a predetermined supersonic speed and enters into the test section. In the test section, the expanded air stream must be about $1000 \mathrm{~K}$ or above to be able to ignite the hydrogen flow. If Mach 2.5 is desired in the test section, the total temperature of air stream required is about $2000 \mathrm{~K}$. If the heating process were to consist only of vitiation, a tremendous amount of hydrogen is required, and the oxidizer stream is diluted by a large amount of water vapor, which the proposed two-stage heating system will help to reduce.

The flow physics inside the test facility of reacting shear layers is complex. It is imperative to know the overall thermodynamic properties of various test conditions to control the experiments. Detailed flow-field prediction of each section of the test rig is also desirable. Since there is no chemical reactions in the low speed flow stream (fuel stream), the current paper is concentrated on the calculations of hot air stream and the mixing layers. The objectives are:

1. Design the nozzle contour to provide a nearly uniform hot air stream.

2. Predict the flow field of air stream in the nozzle with finite rate chemistry.

3. Compare the results of finite rate chemistry with the equilibrium calculations for the hot air stream.

4. Develop the $k-\epsilon$ solver with compressibility effects for the simulation of mixing layers.

\section{THEORETICAL MODEL}

\section{Elow Equations}

The flow equations are taken to be the two dimensional, compressible, Navier-Stokes equations and species transport equations. Written in a strong conservative form, the governing equations can be expressed as follows:

$$
\frac{\partial \mathbf{Q}}{\partial t}+\frac{\partial}{\partial \boldsymbol{x}}\left(\mathbf{E}-\mathbf{E}_{\boldsymbol{v}}\right)+\frac{\partial}{\partial y}\left(\mathbf{F}-\mathbf{F}_{v}\right)=\mathbf{H}
$$

Here $x$ and $y$ are Cartesian coordinates, $\mathrm{Q}$ is the dependent variable, $\mathbf{E}$ and $\mathbf{F}$ are the convective flux vectors:

$$
\begin{gathered}
\mathbf{Q}=\left(\begin{array}{c}
\rho \\
\rho u \\
\rho v \\
\rho e \\
\rho Y_{i}
\end{array}\right), \\
\mathbf{E}=\left(\begin{array}{c}
\rho u \\
\rho u^{2}+p \\
\rho u v \\
u(\rho e+p) \\
\rho u Y_{i}
\end{array}\right), \quad \mathbf{F}=\left(\begin{array}{c}
\rho v \\
\rho u v \\
\rho v^{2}+p \\
v(\rho e+p) \\
\rho v Y_{i}
\end{array}\right)
\end{gathered}
$$

$\mathbf{E}_{v}$ and $\mathbf{F}_{v}$ are the viscous flux vectors:

$$
\begin{aligned}
& \mathbf{E}_{v}=\left(\begin{array}{c}
0 \\
\tau_{x x} \\
\tau_{x y} \\
u \tau_{x x}+v \tau_{x y}-q_{x} \\
-\rho \hat{u}_{i} Y_{i}
\end{array}\right) \\
& \mathbf{F}_{v}=\left(\begin{array}{c}
0 \\
\tau_{y x} \\
\tau_{y y} \\
u \tau_{y x}+v \tau_{y y}-q_{y} \\
-\rho \hat{v}_{i} Y_{i}
\end{array}\right)
\end{aligned}
$$


and $\mathbf{H}$ is the source vector:

$$
\mathbf{H}=\left(\begin{array}{c}
0 \\
0 \\
0 \\
0 \\
\dot{\omega}_{i}
\end{array}\right)
$$

where $i=1, N_{s}-1$. The specific total energy, $e$, shear stress components, $\tau$, and heat flux components, $q$, are given as:

$$
\begin{gathered}
e=\sum_{i=1}^{N_{0}} e_{i}+\frac{1}{2}\left(u^{2}+v^{2}\right) \\
\tau_{x x}=2 \mu \frac{\partial u}{\partial x}-\frac{2}{3} \mu\left(\frac{\partial u}{\partial x}+\frac{\partial v}{\partial y}\right) \\
\tau_{y y}=2 \mu \frac{\partial v}{\partial y}-\frac{2}{3} \mu\left(\frac{\partial u}{\partial x}+\frac{\partial v}{\partial y}\right) \\
\tau_{x y}=\tau_{y x}=\mu\left(\frac{\partial u}{\partial y}+\frac{\partial v}{\partial x}\right) \\
q_{x}=-k \frac{\partial T}{\partial x}+\rho \sum_{i=1}^{N_{s}} h_{i} Y_{i} \hat{u}_{i} \\
q_{y}=-k \frac{\partial T}{\partial y}+\rho \sum_{i=1}^{N_{s}} h_{i} Y_{i} \hat{v}_{i}
\end{gathered}
$$

In the preceding expressions, $\rho$ is the density, $u$ and $v$ are the Cartesian velocity components, $p$ is the pressure, and $e$ is the specific total energy. The subscript $i$ identifies each species, and $N_{s}$ is the total number of species. For the $i$ th species, $Y_{i}, e_{i}, h_{i}$, and $\dot{\omega}_{i}$ are its mass fraction, specific internal energy, enthalpy, and production rate, respectively. The enthalpy of species $i$ is obtained by an integration of $C_{\mathfrak{p}}$ versus temperature:

$$
h_{i}=\int_{0}^{T} C_{p i} d T
$$

where $C_{p i}$ is the constant pressure specific heat which is expressed as a fourth order polynomial of temperature:

$$
C_{p i}=C_{p i 0}+C_{p i 1} T+C_{p i 2} T^{2}+C_{p i 3} T^{3}+C_{p i 4} T^{4}
$$

The internal energy of species $i$ can be obtained from $h_{i}$ using the ideal gas assumption which is valid for high temperature:

$$
e_{i}=h_{i}-R_{i} T
$$

where $R_{i}$ is the gas constant for species $i$. The diffusion velocity components, $\hat{u}_{i}$ and $\hat{v}_{i}$ are calculated by Fick's law ${ }^{4}$ :

$$
\begin{aligned}
& Y_{i} \hat{u}_{i}=-D_{i m} \frac{\partial Y_{i}}{\partial x} \\
& Y_{i} \hat{v}_{i}=-D_{i m} \frac{\partial Y_{i}}{\partial y}
\end{aligned}
$$

where

$$
D_{i m}=\left(1-X_{i}\right) / \sum_{i \neq j} \frac{X_{i}}{D_{i j}}
$$

is the effective binary diffusivity of species $i$ in the gas mixture, and $X_{i}$ is the mole fraction of species i. Transport properties such as the viscosity and the thermal conductivity of each species are considered as polynomials of temperature, and those of the mixture are calculated based on Wilke's mixing rule ${ }^{5}$. The binary mass diffusivities are calculated using ChapmanEnskog theory in conjunction with Lennard-Jones intermolecular potential functions. ${ }^{6}$

\section{Two-Equation Turbulence Model}

In deriving the $k$ and $\epsilon$ equations, all the flow properties are taken Favre averaged (mass weighted averaged) except the density $(\rho)$. The definition of the Favre average is $\tilde{\phi}=\bar{\phi} \rho / \bar{\rho}$ where $\phi$ is any flow property. In doing so, the resulting equations are much simpler because all the terms associating with the density fluctuation, e.g. $\overline{\rho^{\prime} u^{\prime}}$, were eliminated. Thus, Whenever the $k-\epsilon$ model is applied, all the variables in the aforementioned equation are changed to the mass averaged variables, and the transport properties are changed to the effective, i.e. laminar plus turbulent, properties. For convenience, no additional flow equations for turbulent flows with the two equation model is elaborated here.

The $k$ and $\epsilon$ equations in the Cartesian coordinate system can be cast into the vector form:

$$
\frac{\partial \mathbf{Q}_{k e}}{\partial t}+\frac{\partial \mathbf{E}_{k e}}{\partial x}+\frac{\partial \mathbf{F}_{k e}}{\partial y}=\frac{\partial \mathbf{E}_{v k e}}{\partial x}+\frac{\partial \mathbf{F}_{v k e}}{\partial y}+\mathbf{S}
$$

where

$$
\begin{gathered}
\mathbf{Q}_{k e}=\left(\begin{array}{c}
\rho k \\
\rho \epsilon
\end{array}\right) \\
\mathbf{E}_{k e}=\left(\begin{array}{c}
\rho U k \\
\rho U \epsilon
\end{array}\right) \\
\mathbf{F}_{k e}=\left(\begin{array}{c}
\rho V k \\
\rho V \epsilon
\end{array}\right) \\
\mathbf{E}_{v k e}=\left(\begin{array}{c}
\frac{\mu_{\epsilon}}{\sigma_{k}} \frac{\partial k}{\partial x} \\
\mu_{\xi} \frac{\partial \epsilon}{\partial x}
\end{array}\right) \\
\mathbf{F}_{v k e}=\left(\begin{array}{c}
\frac{\mu_{1}}{\sigma_{k}} \frac{\partial k}{\partial y} \\
\frac{\mu_{1}}{\sigma_{e}} \frac{\partial \epsilon}{\partial y}
\end{array}\right) \\
\mathbf{S =}\left(\begin{array}{c}
G-\rho \epsilon\left(1+\alpha M_{t}^{2}\right) \\
\left(C_{1} G-C_{2} \rho \epsilon\right) \frac{\epsilon}{k}
\end{array}\right)
\end{gathered}
$$


where $G$ is the turbulence generation term of the $k$ equation and can be expressed as:

$$
G=\mu_{t}\left[2\left(\frac{\partial U^{2}}{\partial x}+\frac{\partial V^{2}}{\partial y}\right)+\left(\frac{\partial U}{\partial y}+\frac{\partial V}{\partial x}\right)^{2}\right]
$$

The eddy viscosity $\mu_{t}$ required by the flow equations is modelled as:

$$
\mu_{t}=C_{\mu} \rho \frac{k^{2}}{\epsilon}
$$

The constants used in the $k-\epsilon$ model are the standard Jones and Launder's values: ${ }^{7} C_{\mu}=0.09, C_{1}=1.44$, $C_{2}=1.92, \sigma_{k}=1.0$, and $\sigma_{\varepsilon}=1.3$. These constants were never altered during the course of this work.

In the source term of the $k$ equation, the dissipation term $\rho \epsilon$ is multiplied by a correction factor $\left(1+\alpha M_{t}^{2}\right)$ to accommodate the compressibility effect. Here $M_{t}$ is the local turbulence $M$ ach number defined as $M_{t}=\sqrt{k} / a$ where $a$ is the local speed of sound. The constant $\alpha$ in the term is taken as unity. This model is developed by Sarkar et al. ${ }^{8}$ The physical meaning of the term is that for high turbulence Mach number $\left(M_{t}\right)$ flows, the dissipation of the turbulence kinetic energy is enhanced by a factor of $\alpha M_{t}^{2}$. For free shear layers at high convective Mach number, this correction factor reaches a value of 1.3. Thus, the turbulence intensity is greatly reduced due to this compressibility effect.

In calculating the turbulent frce shear layers, the inlet boundary conditions for mean velocities and temperature are specified based on the hyperbolic tangent profile with specified initial shear layer thickness. The hyperbolic tangent profile is an approximation of the self-similar solution for fully developed turbulent free shear layers. The inlet transverse velocities are set to be zero. The turbulent kinetic energy and dissipation are specified according to the local equilibrium assumption and a algebraic turbulence model: ${ }^{9}$

$$
\mu_{t}=\rho l_{m}^{2} \frac{\partial U}{\partial y}
$$

where $l_{m}=0.125 b$ and the shear layer thickness, $b$, is based on the distance between the two transverse locations where $U=U_{1}-0.1 \Delta U$ and $U=U_{2}+0.1 \Delta U$. The dissipation can be related to the local length scale which is specified based on the local shear layer thickness:

$$
\epsilon=C_{\epsilon} \frac{k^{\frac{3}{2}}}{b}
$$

where $C_{t}=1.23$. Using Eqs. (18) and (19), and the equation for the eddy viscosity, i.e, Eq. (17), $k$ and $\epsilon$ can be readily obtained for the upstream boundary conditions.

\section{Numerical Method}

The numerical solution of Eq. (1) is performed in a general, body-fitted coordinate system, $(\xi, \eta)$. Coordinate transformation of Eq. (1) gives

$$
\frac{\partial \hat{\mathbf{Q}}}{\partial \tau}+\frac{\partial}{\partial \boldsymbol{\xi}}\left(\hat{\mathbf{E}}-\hat{\mathbf{E}}_{v}\right)+\frac{\partial}{\partial \eta}\left(\hat{\mathbf{F}}-\hat{\mathbf{F}}_{v}\right)=\hat{\mathbf{H}}
$$

where

$$
\begin{aligned}
\hat{\mathbf{Q}} & =h \mathbf{Q} \\
\hat{\mathbf{E}} & =h\left(\xi_{x} \mathbf{E}+\xi_{y} \mathbf{F}\right) \\
\hat{\mathbf{F}} & =h\left(\eta_{x} \mathbf{E}+\eta_{y} \mathbf{F}\right) \\
\hat{\mathbf{E}}_{v} & =h\left(\xi_{x} \mathbf{E}_{v}+\xi_{y} \mathbf{F}_{v}\right) \\
\hat{\mathbf{F}}_{v} & =h\left(\eta_{x} \mathbf{E}_{v}+\eta_{y} \mathbf{F}_{v}\right) \\
\hat{\mathbf{H}} & =h \mathbf{H}
\end{aligned}
$$

in which $h$ is the cell volume.

The transformed equation, Eq. (20), is solved using a time-marching, LU scheme. The LU scheme can be obtained by approximately factorizing the left-handside (LHS) of the equation. In time-marching form, the implicit upwind difference scheme of Eq. (20) can be written as

$$
\begin{aligned}
& {\left[\mathbf{I}+\Delta t\left(D_{\xi}^{+} \hat{\mathbf{A}}^{-}+D_{\eta}^{+} \hat{\mathbf{B}}^{-}-\hat{\mathbf{D}}+\right.\right.} \\
& \left.\left.D_{\xi}^{-} \hat{\mathbf{A}}^{+}+D_{\eta}^{-} \hat{\mathbf{B}}^{+}\right)\right] \Delta \hat{\mathbf{Q}}=\Delta t R H S
\end{aligned}
$$

In Eq. (21), $\Delta t$ is the time-step. Backward-difference operators are denoted by $D_{\xi}^{-}$and $D_{\eta}^{-}$, and forwarddifference operators by $D_{\xi}^{+}$and $D_{\eta}^{+}$. The flux Jacobians, $\hat{\mathbf{A}}^{+}, \hat{\mathbf{B}}^{+}, \hat{\mathbf{A}}^{-}$, and $\hat{\mathbf{B}}^{-}$are constructed such that the eigenvalues of ' + ' matrices are nonnegative and those of '-' matrices are nonpositive. The matrix $\hat{\mathbf{D}}$ is the source Jacobian.

The left-hand-side matrix of Eq. (21) is usually too large for direct inversion. An approximate-factorization procedure is implemented to result in the following $\mathrm{LU}$ scheme :

$$
\begin{aligned}
& {\left[\mathrm{I}+\Delta t\left(D_{\xi}^{-} \hat{\mathbf{A}}^{+}+D_{\eta}^{-} \hat{\mathbf{B}}^{+}-\frac{\hat{\mathbf{A}}^{-}}{\Delta \xi}-\frac{\hat{\mathbf{B}}^{-}}{\Delta \eta}-\hat{\mathbf{D}}\right)\right]} \\
& \left(\mathrm{I}+\frac{\Delta t}{\Delta \xi}\left(\hat{\mathbf{A}}^{+}-\hat{\mathbf{A}}^{-}\right)+\frac{\Delta t}{\Delta \eta}\left(\hat{\mathbf{B}}^{+}-\hat{\mathbf{B}}^{-}\right)\right)^{-1} \\
& {\left[\mathrm{I}+\Delta t\left(D_{\xi}^{+} \hat{\mathbf{A}}^{-}+D_{\eta}^{+} \hat{\mathbf{B}}^{-}+\frac{\hat{\mathbf{A}}^{+}}{\Delta \xi}+\frac{\hat{\mathbf{B}}^{+}}{\Delta \eta}\right)\right] \Delta \hat{\mathbf{Q}}} \\
& =\Delta t R H S
\end{aligned}
$$

in which the grid spacing in the general coordinate, $\Delta \xi$ and $\Delta \eta$ are usually taken to be one. Equation (22) is 
the generic form for the LU schemes. Its derivation can be found in Ref. 10 and will not be repeated here. This LU scheme requires inversion of the matrix,

$$
\left[\mathbf{I}+\Delta t\left(\hat{\mathbf{A}}^{+}-\hat{\mathbf{A}}^{-}+\hat{\mathbf{B}}^{+}-\dot{\mathbf{B}}^{-}-\hat{\mathbf{D}}\right)\right]
$$

for the L operator and

$$
\left[\mathrm{I}+\Delta t\left(\hat{\mathbf{A}}^{+}-\hat{\mathbf{A}}^{-}+\hat{\mathbf{B}}^{+}-\hat{\mathbf{B}}^{-}\right)\right]
$$

for the $U$ operator. Note that the source Jacobian appears in the L operator only. This is intentionally designed to minimize computing the source Jacobian, which is quite expensive for most non-equilibrium chemistry models.

Up to this point, no definition has been made to the exact form of the split flux Jacobians. For LUSSOR, the split flux Jacobians are defined as

$$
\begin{aligned}
& \hat{\mathbf{A}}^{+}=0.5\left(\hat{\mathbf{A}}+\gamma_{\dot{\mathbf{A}}} \mathrm{I}\right) \\
& \hat{\mathbf{A}}^{-}=0.5\left(\hat{\mathbf{A}}-\gamma_{\dot{\mathbf{A}}} \mathbf{I}\right) \\
& \hat{\mathbf{B}}^{+}=0.5\left(\hat{\mathbf{B}}+\gamma_{\dot{\mathbf{B}}} \mathbf{I}\right) \\
& \hat{\mathbf{B}}^{-}=0.5\left(\hat{\mathbf{B}}-\gamma_{\dot{\mathbf{B}}} \mathbf{I}\right)
\end{aligned}
$$

where $\gamma_{\hat{\mathbf{A}}}$ and $\gamma_{\hat{\mathbf{B}}}$ are greater than the spectral radii of the associated flux Jacobians :

$$
\begin{aligned}
& \gamma_{\hat{\mathbf{A}}} \geq \max \left(\left|\lambda_{\dot{\mathbf{A}}}\right|\right) \\
& \gamma_{\hat{\mathbf{B}}} \geq \max \left(\left|\lambda_{\dot{\mathbf{B}}}\right|\right)
\end{aligned}
$$

The purpose of constructing split flux-Jacobians by Eq. (24) is to make the matrices in Eqs. (23.a) and (23.b) diagonal for efficient inversion. Apparently, the eigenvalues of the split flux-Jacobians are not the characteristics speeds of the flow.

In deriving Eq. (23) for the LU-SSOR the equivalent $\gamma$ is assumed to be frozen. This assumption tremendously simplifies the algebra involved, and curtails the computational operations needed.

In calculating the RHS, both the inviscid and viscous terms are discretized using the central-difference approach:

$$
R H S=D_{\xi}\left(\hat{\mathbf{E}}_{v}-\hat{\mathbf{E}}\right)+D_{\eta}\left(\hat{\mathbf{F}}_{v}-\hat{\mathbf{F}}\right)+\hat{\mathbf{H}}
$$

where $D_{\xi}$ and $D_{\eta}$ are the central difference operators.

In solving the $k$ and $\epsilon$ equations, the aforementioned numerical method, i.e., the LU-SSOR on the left hand side and central differencing on the right hand side, is used. The solution procedure of the whole equation set is decoupled into flow solver and turbulence solver. Thus, the turbulence solver stands alone and can be easily turn on or off. This arrangement does not affect the overall numerical stability due to the fact that the feedback from $k$ and $\epsilon$ equations to flow equations depends on turbulent transport properties only. Thus, it is more efficient and convenient to separate the solution procedure into two parts.

The source terms of the $k$ and $\epsilon$ equations demand special treatment. In linearizing the source terms for the numerical method, Jacobian matrix is obtained through the derivative of the source terms with respect to the dependent variables, i.e., $\rho k$ and $\rho \epsilon$. Following the usual practice, the form of the source terms guarantee a $2 \times 2$ full matrix for the Jacobian matrix. However, special treatment in deriving the Jacobian matrix is applied in this work to enhance the numerical stability. In the $k$ equation, $\epsilon$ has been replaced by $k(\epsilon / k)$ where $\epsilon / k$ is treated as a constant. A similar method is also applied to the source term of $\epsilon$ equation. The Jacobian matrix obtained is:

$$
\mathbf{D}=\left(\begin{array}{cc}
-\frac{\epsilon}{k}\left(1+\alpha M_{t}^{2}\right) & 0 \\
0 & -C_{2} \frac{\epsilon}{k}
\end{array}\right)
$$

Note that off-diagonal terms are eliminated and the diagonal terms are always negative. Thus, the implicit part of the source terms of $k$ and $\epsilon$ equations behaves like a sink which always stabilize the numerical scheme.

\section{Results and Discussions}

\section{Equilibrium Calculations}

The chemical equilibrium program (CET89 code) developed by Gordon and McBride ${ }^{11}$ at the NASA Lewis Center is adopted for the overall evaluation of air stream conditions. By providing initial condition of the oxidizer and fuel, the CET89 code performs a free energy minimization to obtain chemical composition and thermodynamic properties at equilibrium status. $\Lambda \mathrm{d}$ ditionally, it also provides isentropic expansion calculations for either frozen or equilibrium processes. Since we are interested in the thermodynamic properties of the air stream after expansion, this special feature of the CET89 code, namely, the rocket performance calculation, is used.

Table 1 shows a typical result of the equilibrium calculation with isentropic expansion. The first part of the table shows the initial condition of the flow system: one mole of air at $860 \mathrm{~K}$ mixed with hydrogen and oxygen at about $300 \mathrm{~K}$. The total pressure is 17 atm, and hydrogen and oxygen is in stoichiometric ratio for vitiation heating process. This is a H-P problem that specifies the total enthalpy and and total pressure of the system and then calculates the adiabatic flame 
temperature. Iterative calculations adjusting the the amount of hydrogen and oxygen added to the system yields the desired temperature and Mach number of the expanded air flow. As shown in Table 1, for one mole of air, 0.22 mole of hydrogen and 0.11 mole of oxygen are needed for vitiation heating to obtain a hot air stream at Mach 2.5 and $1000 \mathrm{~K}$ of temperature.

Equilibrium calculations are used to account for the changing species composition during the expansion process before the air enters the test section. Inside the combustor chamber, minor species such as $\mathrm{HO}_{2}, \mathrm{NO}$, $\mathrm{OH}^{-. . . e t c}$. exists. During the expansion process, these high energy radicals recombine and form more stable species such as $\mathrm{H}_{2} \mathrm{O}, \mathrm{O}_{2}$, and $\mathrm{N}_{2}$; according to calculation, no minor species survives. During the expansion, specific heat of constant pressure $\left(C_{p}\right)$ and specific heat ratio $(\gamma)$ change significantly due to the change of the species composition and the diminishing of the vibration mode of internal energy. Therefore, the so called "real gas effect" must be included in the CFD simulation of the flow expansion.

While the equilibrium calculation has provided a global understanding of the vitiation and expansion processes of the air stream, a more detailed flow field solution is invaluable for the understanding of the two dimensional effect, boundary layer flow, and the finite rate flow chemistry.

\section{Finite-Rate Calculations}

Before the Navier Stoke calculation, the nozzle contour for the flow acceleration is designed. A commercially available MOC (Method Of Characteristics) code based on Rao's method is adopted in designing the nozzle contour. The calculation is based on the ideal gas with constant specific heat ratio. The code performs wave cancellation calculation to deduce the nozzle contour which provides a uniform flow at the exit of the nozzle. However, due to the existence of a contoured center body in the experiment, the designed contour can not be applied to the test rig directly. As shown in Fig. 3, the subsonic part of the nozzle must be bent. Therefore, CFD code is needed to verify that the bent nozzle still provide a uniform flow into the test section. Figure 3 shows the geometry of a Mach 2.5 nozzle for the shear layer facility. The contour of the nozzle is designed using the Rao code. The subsonic part of the nozzle is bent to accommodate the center body which separates the high speed stream and low speed stream.

The rest of the figures shows calculations of the RPLUS code. Figure 4 shows the Mach number contour for the supersonic bent nozzle. The Mach number at the exit is about 2.4. The fluid accelerates dramatically in the throat region and becomes supersonic in the diverging part of the nozzle. Due to the high shear stresses, the boundary layers in the supersonic part of the nozzle are significantly thicker that that in the subsonic region. Figure 5 shows the Mach number profile at the exit of the nozzle (the inlet of the test section of shear layers). Except for the existence of boundary layers, the flow is quite uniform at the nozzle exit. Thus, the bent nozzle did not aggravate the uniform flow designed by the MOC calculation. The temperature contour is shown in Fig. 6. Temperature decreases as the flow accelerates. Note that the temperature at the nozzle exit is about $1100 \mathrm{~K}$, which compares favorably with the equilibrium calculation, theoretically, this temperature is adequate for the ignition of diluted hydrogen flow provided by another stream. Again, strong temperature gradient occurred inside the boundary layers due to high shear stress. The pressure contour is illustrated in Fig. 7 showing that the pressure decreases as the flow speeds up. The pressure at the inlet of the test section is about 1 atm. This test condition of relative lower pressure curtails complex treatment of the hydrogen/air combustion chamber. The pressure predicted by flow solver also agrees favorably with that of the equilibrium calculation.

Figures 8-11 show the species concentration contours of $\mathrm{H}_{2}, \mathrm{O}_{2}, \mathrm{H}_{2} \mathrm{O}$, and $\mathrm{OH}$. The chemical kinetics is the dominant factor in determining the species distribution and kinetics is strongly temperature dependent. Thus, the pattern of the species contours resemble that of temperature, i.e., very little change in the subsonic region and significant recombination in the supersonic region. Basically, very minor change occurs for the major species such as $\mathrm{O}_{2}, \mathrm{H}_{2}$, and $\mathrm{H}_{2} \mathrm{O}$, whereas high energy radicals, such as $\mathrm{OH}$, diminished in the flow acceleration process. The mass fraction of oxygen at the exit of the nozzle is about $20 \%$, which is very close to the composition of air. The vitiation system using oxygen and hydrogen does provide desirable oxidizer stream.

\section{Turbulent Shear Laver Calculations}

The test case of the newly developed $k-\epsilon$ solver is one of the experimental tests reported by Dutton et al. ${ }^{3}$ The test condition is tabulated as Table 2 . In this case, two streams at different temperatures, densities, and Mach numbers merge together to form a nonreacting free shear layer. The convective Mach number is 0.45 . Figure 12 shows the development of the free shear layer. Note that $x$ and $y$ axes are not on 1:1 ratio for the convenience of observation. The definition of the shear layer boundaries is the same that of the shear layer thickness. In Fig. 12, the boundaries of 
the shear layer corresponding to $10-90 \%$ are drawn. Circles are the experimental data by Dutton et al. The calculated results underpredict the development of the shear layer. After the developing region, the boundary of the shear layer is almost linear. Incidentally, Fig. 13 shows the numerical convergence trends of the flow and $k-\epsilon$ equations. In about 2500 iterations, the residuals drop about 12 orders of magnitude and reach the machine accuracy.

Figure 14 shows the Mach number profile at various axial locations. The velocity gradient in the transverse direction decreases as the flow goes downstream. Figure 15 is the coalesced version of Fig. 14. The nondimensionalized y coordinate defined as $\left(y-y_{c}\right) / \delta$ is used, where $y_{c}$ is the transverse location at the center of the shear layer, and $\delta$ is the shear layer thickness. Note that the upstream boundary condition of velocities is prescribed according to a hyperbolic tangent curve which is an approximation of self-similar solution of free shear layers. According to Fig. 15, this self similarity of velocity profiles never fail as the flow goes downstream.

Figure 16 shows coalesced turbulence kinetic energies at various locations. The turbulence kinetic energy is nondimensionalized by $\Delta U^{2}$. This figure clearly shows that after the first three stations, i.e., about 150 $\mathrm{mm}$, the turbulence kinetic energy retains self similarity. Thus, the developing region for the turbulence is about $150 \mathrm{~mm}$. Figure 17 shows the turbulence dissipation profiles. As flow goes downstream, the peak values of turbulence dissipation at each stations decrease, and the turbulence dissipation never reach a fully developed condition. However, if the $\epsilon$ value is nondimensionalized by $\Delta U^{3} / \delta$ the coalesced profiles appear as shown in Fig. 17. Similar characteristic is observed in the eddy viscosity profiles (Fig. 18). Figure 19 shows the nondimensionalized Reynolds stress profiles. Again, the turbulence Reynolds stress reach the fully developed condition at about $150 \mathrm{~mm}$ downstream of the splitter plate.

Figure 20 shows the comparison between the predicted fully developed Reynolds stress and the experimental data reported by Dutton et al. ${ }^{3}$ The predicted solution underestimated the peak value of the Reynolds stress profile by $6 \sim 8 \%$; however, the overall trend of the predicted results is correct. Many reasons contribute to the discrepancy between the predicted result and experimental data. Among them, the upstream boundary conditions are simplified in the solution procedure, i.e., no effort was made to simulate two boundary layers merging at the tip of the splitter plate. This could offset the solution in the leveloping region and shift the fully developed solutions.

\section{CONCLUDING REMARKS}

CFD application to the design of the facility lies in the provision of detailed information of the whole flow field. The chemical reacting flow solver (RPLUS2D) is applied in the design process of a compressible free shear layer facility. However, a one-dimensional equilibrium calculation is an indispensable tool for the overall design of the reacting shear system. The thermodynamic properties predicted by the equilibrium calculation can serve as a yard stick for detailed flow field calculations. A converging-diverging nozzle is used to provide a supersonic stream as the high speed stream of the shear layer facility. Detailed analyses are performed to study the flow and chemical characteristics of the nozzle flows. The chemistry in the subsonic region of the nozzle flow is largely equilibrium and little change of chemical composition is observed with all high energy radicals recombining in the supersonic region.

A $k-\epsilon$ solver with compressibility effect is added into the RPLUS2D code. A nonreacting turbulent shear layer is simulated by the newly developed program. Preliminary results show favorable comparison with Dutton's experimental data.

\section{REFERENCES}

1. N.T. Clemens and M.G. Mungal, "Two and ThreeDimensional Effects in the Supersonic Mixing Layer," AIA A Paper 90-1978, July 1990.

2. J. Hall and P. Dimotakis, "The Supersonic Hydrogen Fluorine Combustion Facility design review report," GALTIC Internal Report, 1989.

3. S.G. Goebel and J.C. Dutton, "Velocity Measurement of Compressible, Turbulent Mixing Layers," AIAA Paper 90-0709, January 1990.

4. K.K. Kuo Principles of Combustion, p. 165. John Wiley \& Sons, Inc., New York, 1986.

5. C.R. Wilke, "A Viscosity Equation for Gas Mixture," J. Chem. Phys., Vol. 18, No. 4, Apr. 1950, p. 517.

6. R.C. Reid, J.M. Prausnitz, and T.K. Sherwood, The Properties of Gases and Liquids, 3rd Ed., McGraw-Hill, New York, 1987.

7. W.P. Jones and B.E. Launder, "The Prediction of Laminarization with a Two-Equation Model of Turbulence," Int. J. Heat Mass Transfer, Vol. 15, 1972 , p. 301.

8. S. Sarkar, G. Erlebacher, M.Y. Hussaini, and H.O. Kreiss, "The Analysis and Modeling of Dilatational Terms in Compressible Turbulence," ICASE 
Report No. 89-79, NASA Langley Research Center, 1989.

9. R.F. Burr and J.C. Dutton, "Numerical Modeling of Compressible Reacting Turbulent Shear Layers," AIA A Paper 90-1463, June 1990.

10. S.T. Yu, P.Y. Tsai, and J.S. Shuen, "ThreeDimensional Calculation of Supersonic Reacting
Flows Using an LU Scheme," AlA A paper 89-0391, January 1989.

11. S. Gordon and B.J. McBride, "Computer Program for Calculation of Complex Chemical Equilibrium Compositions, Rocket Performance, Incident and Reflected Shocks, and Chapman-Jouguet Detonations," NASA SP-273, 1973.

Table 1: Results of equilibrium calculation for Mach 2.5 oxidizer stream.

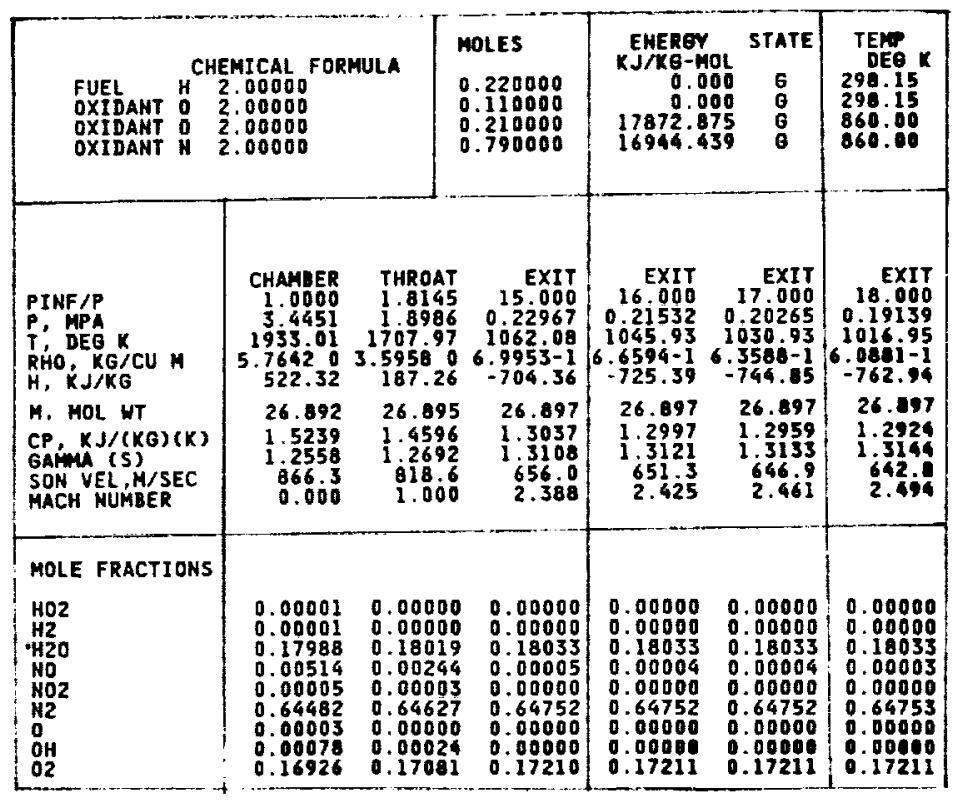

Table 2: Test conditions of the calculated shear layer.

\begin{tabular}{|l|l|l|}
\hline Item & Units & Value \\
\hline $\mathrm{M}_{1}, \mathrm{M}_{2}$ & & $1.91,1.37$ \\
$\mathrm{M}_{\mathrm{c}}$ & & 0.45 \\
$\mathrm{U}_{1}, \mathrm{U}_{2}$ & {$[\mathrm{~m} / \mathrm{s}]$} & 702,404 \\
$\mathrm{~T}_{1}, \mathrm{~T}_{2}$ & {$[\mathrm{~K}]$} & 334,215 \\
$\mathrm{P}_{1}, \mathrm{Q}_{2}$ & {$\left[\mathrm{~K} \mathrm{~g} / \mathrm{m}^{3}\right]$} & $0.57,0.89$ \\
$\mathrm{P}^{\prime}$ & {$[\mathrm{atm}]$} & 0.55 \\
$\delta^{\prime}$ & & 0.027 \\
$\delta^{\prime} / 8^{\prime}$ & & 0.54 \\
\hline
\end{tabular}




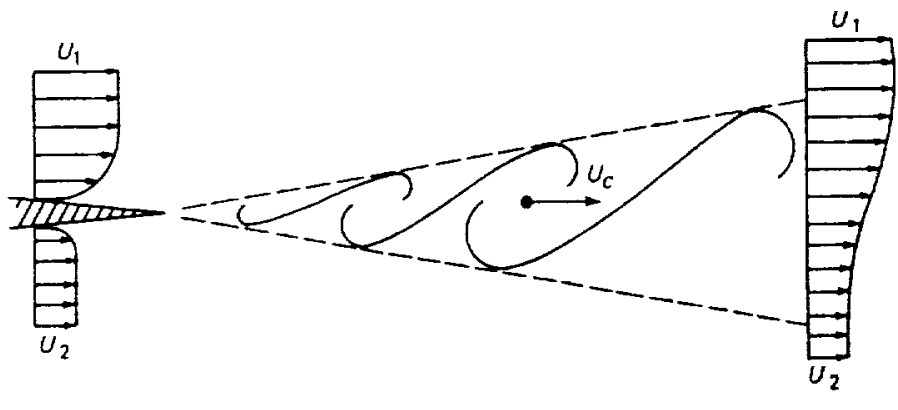

Fig. 1: Schematic of free shear layer.

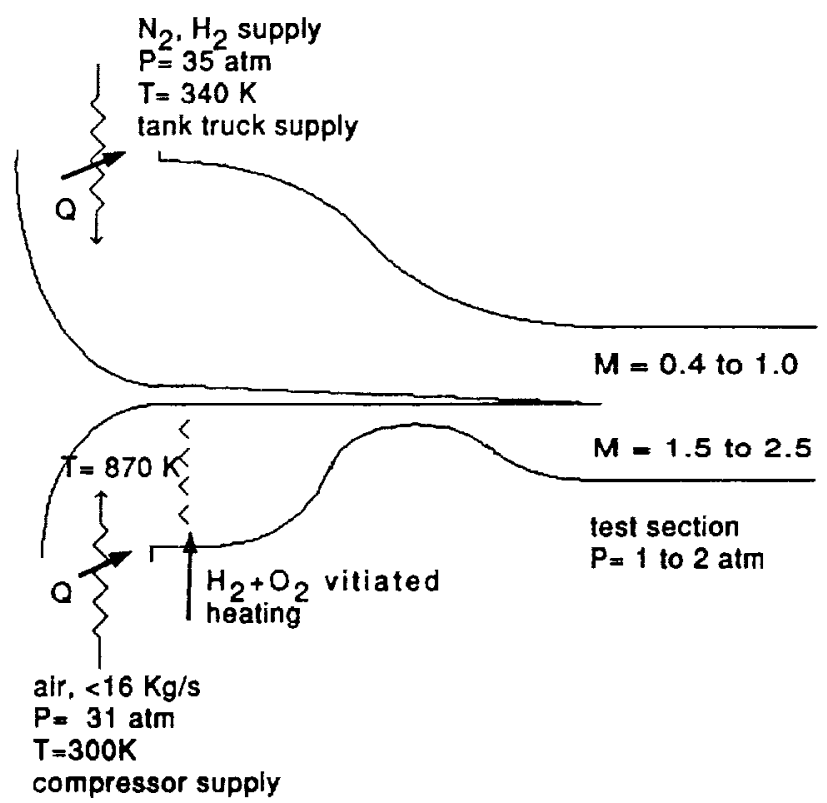

Fig. 2: NASA supersonic shear layer test facility.

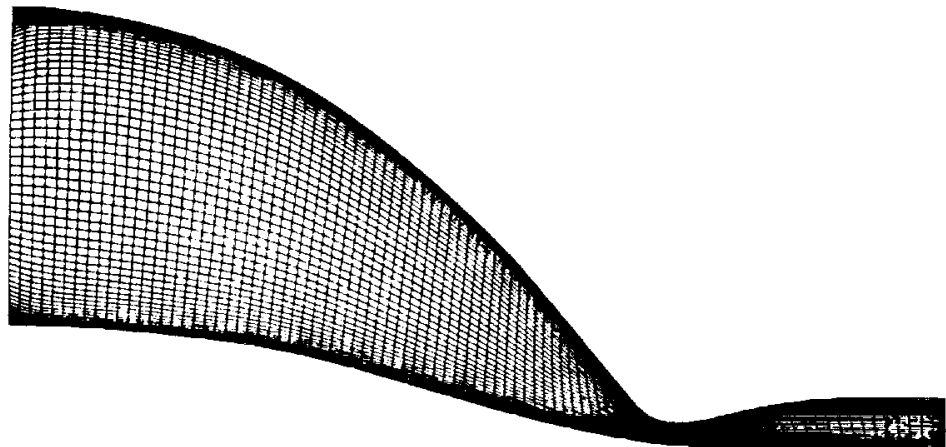

Fig. 3: Numerical grid for the Mach 2.5 nozzle (grid size $90 \times 70)$.

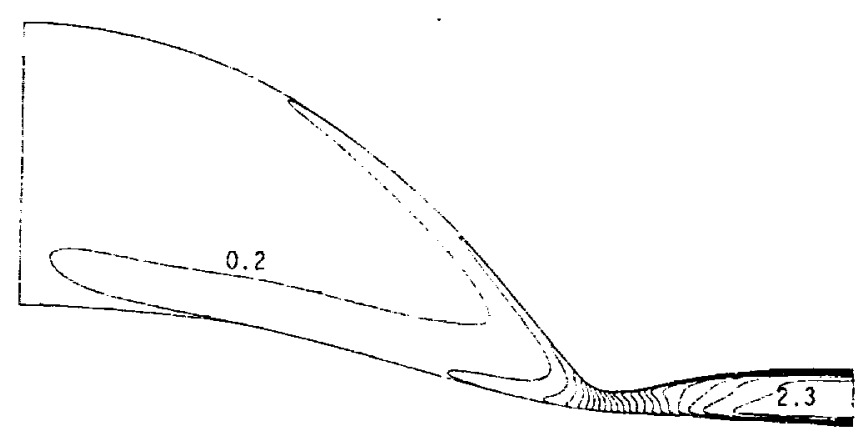

Fig. 4: Mach number contours of the Mach 2.5 nozzle.

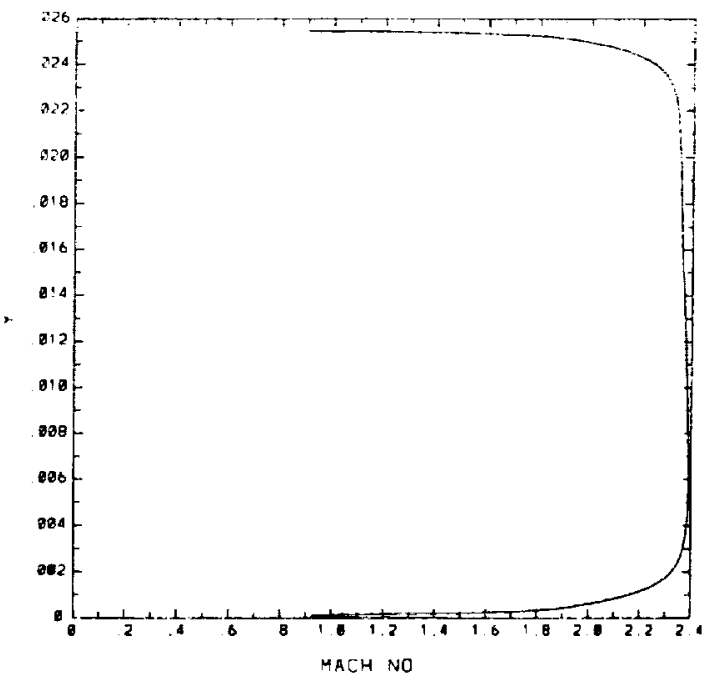

Fig. 5: Mach number profile at the nozzle exit.

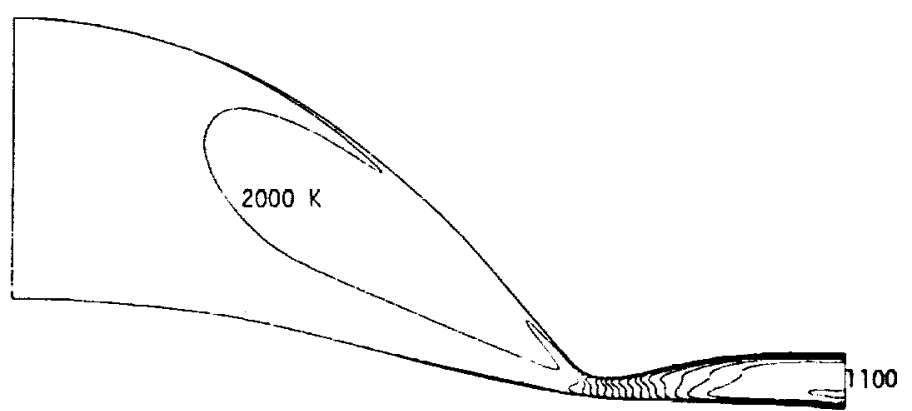

Fig. 6: Temperature contours of the Mach 2.5 nozzle. 


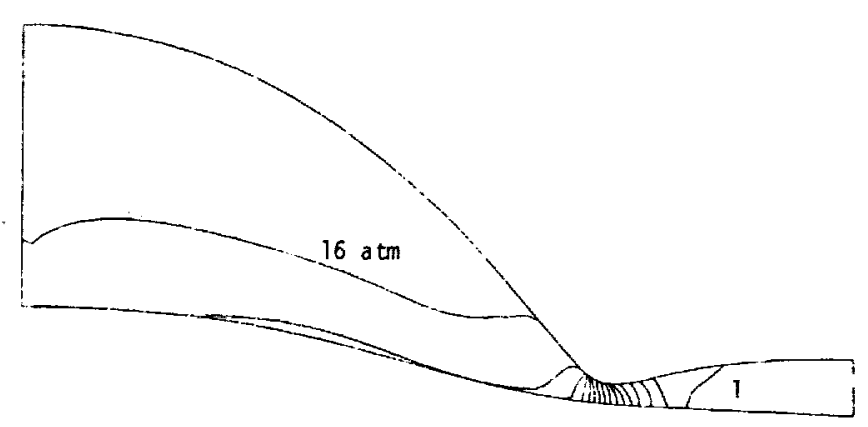

Fig. 7: Pressure contours of the Mach 2.5 nozzle.

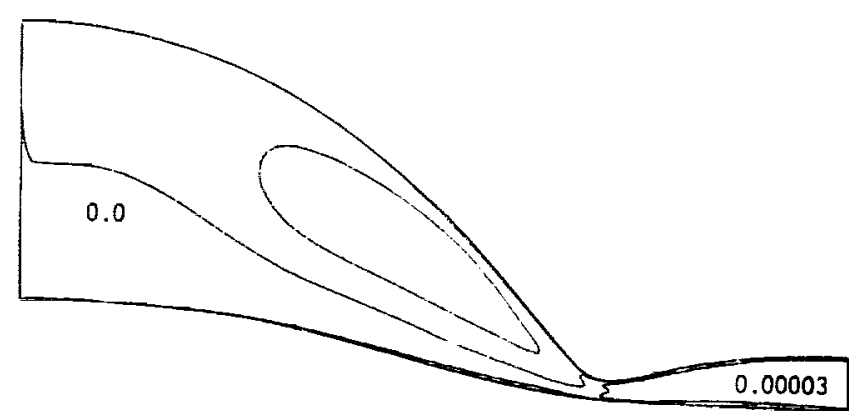

Fig. 8: $\mathrm{H}_{2}$ mass fraction contours of the Mach 2.5 nozzle.

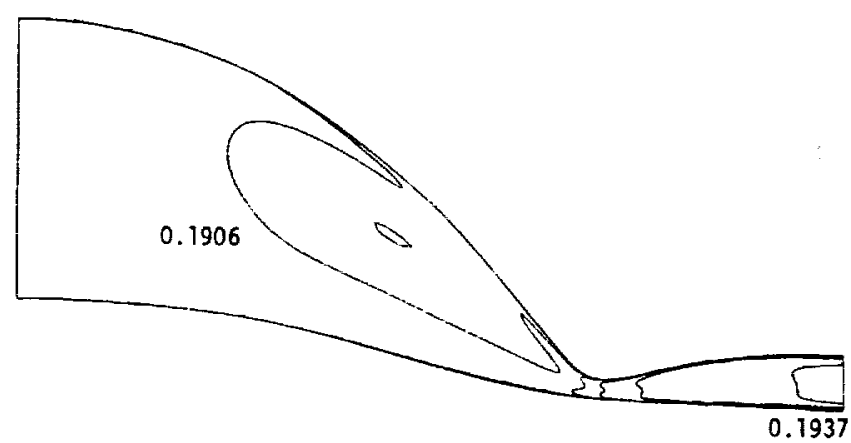

Fig. 9: $\mathrm{O}_{2}$ mass fraction contours of the Mach 2.5 nozzle.

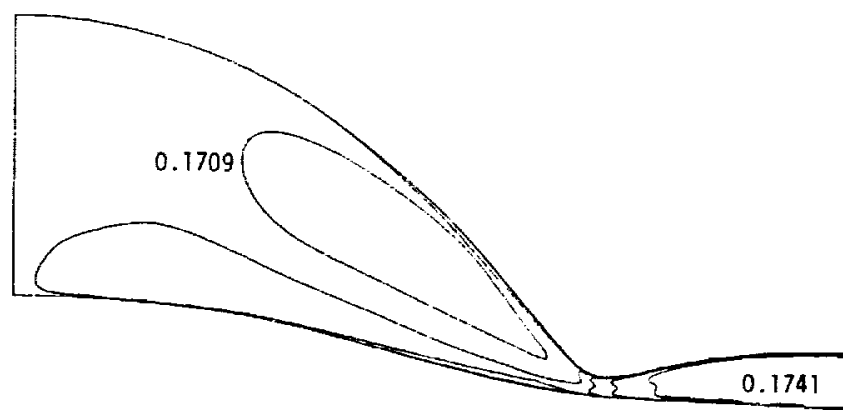

Fig. 10: $\mathrm{H}_{2} \mathrm{O}$ mass fraction contours of the Mach 2.5 nozzle.

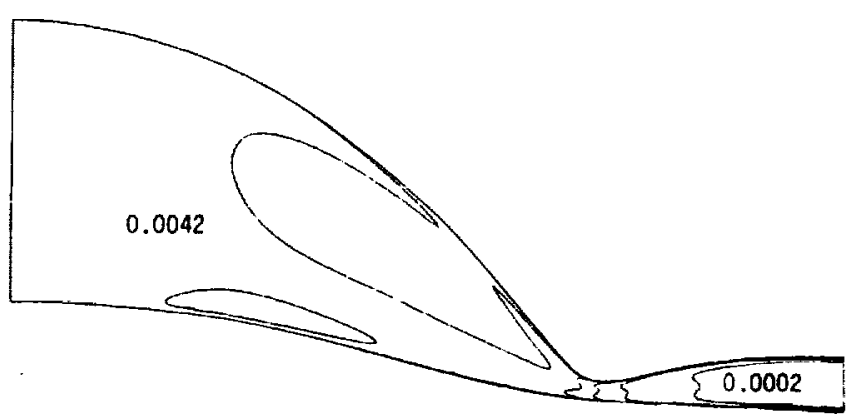

Fig. 11: OH mass fraction contours of the Mach 2.5 nozzle. 


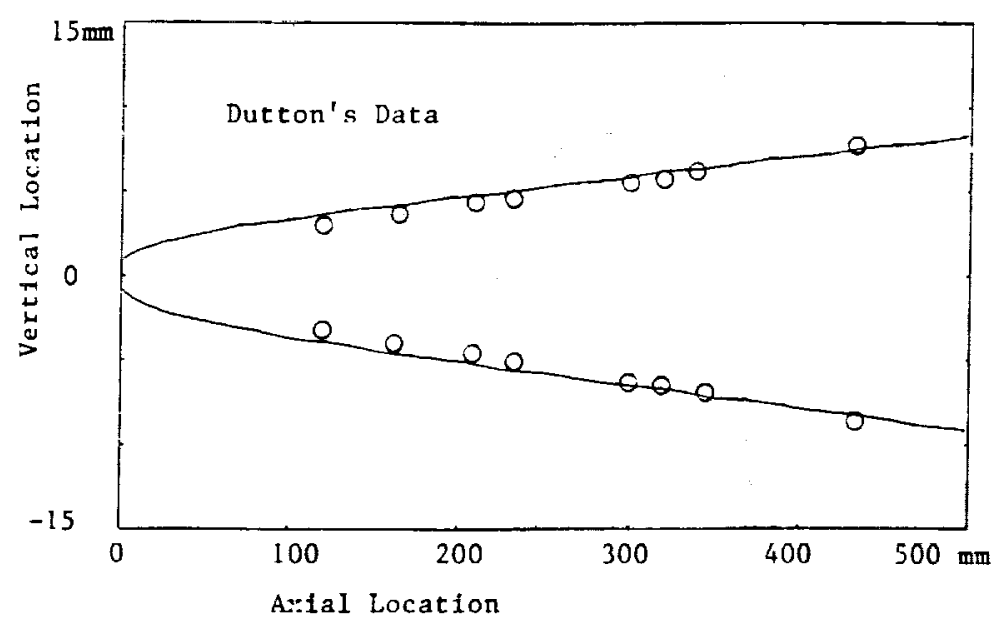

Fig. 12: The boundaries of the free shear layer (10--90\%).
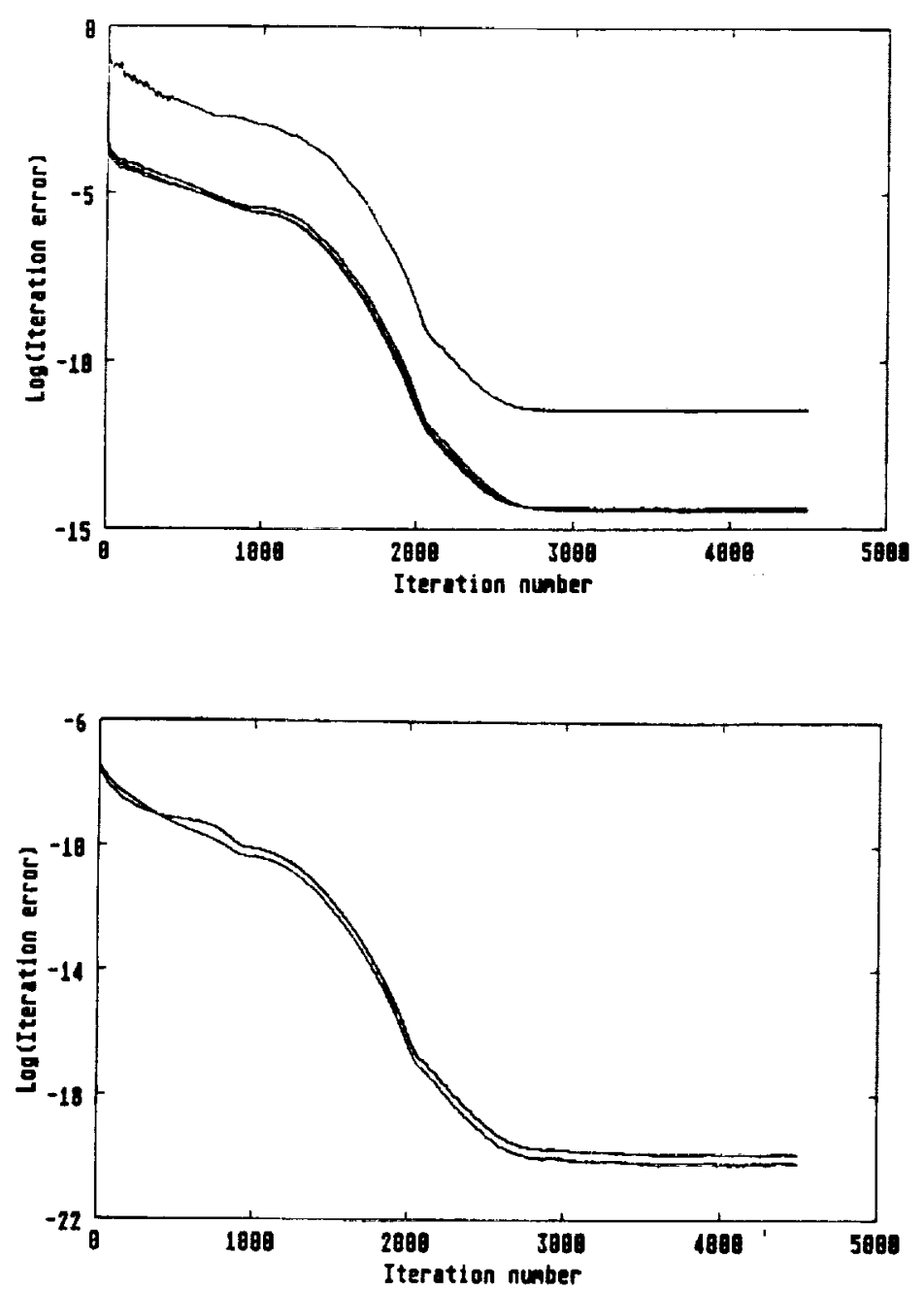

Fig. 13: Convergence trends of the flow and turbulence equations.

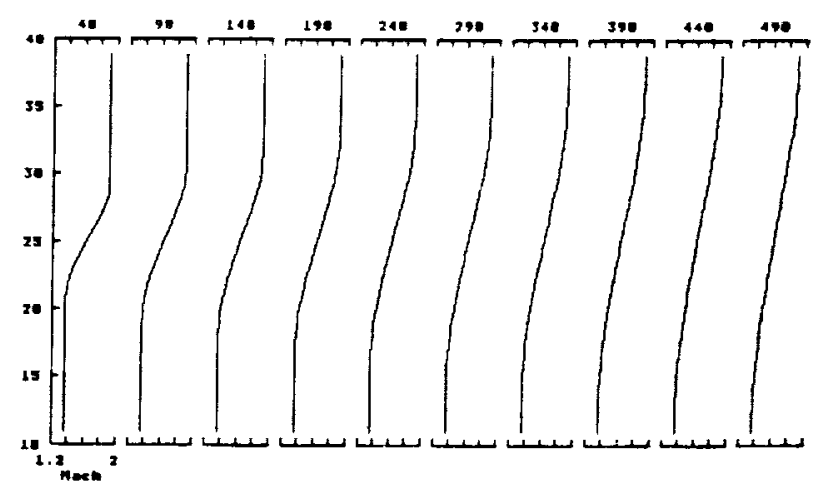

Fig. 14: The velocity profiles of the free shear layer at different axial locations.
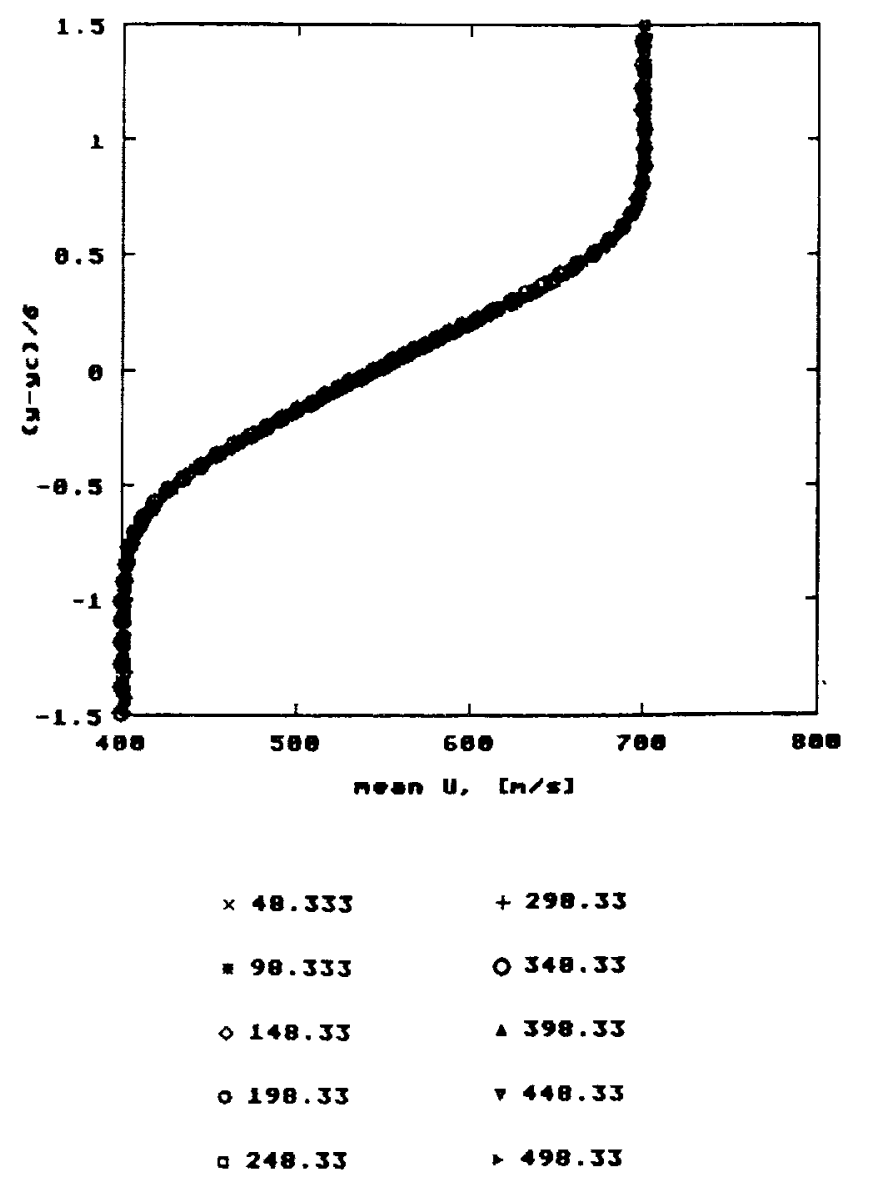

Fig. 15: Coalesced velocity profiles of different axial locations. 


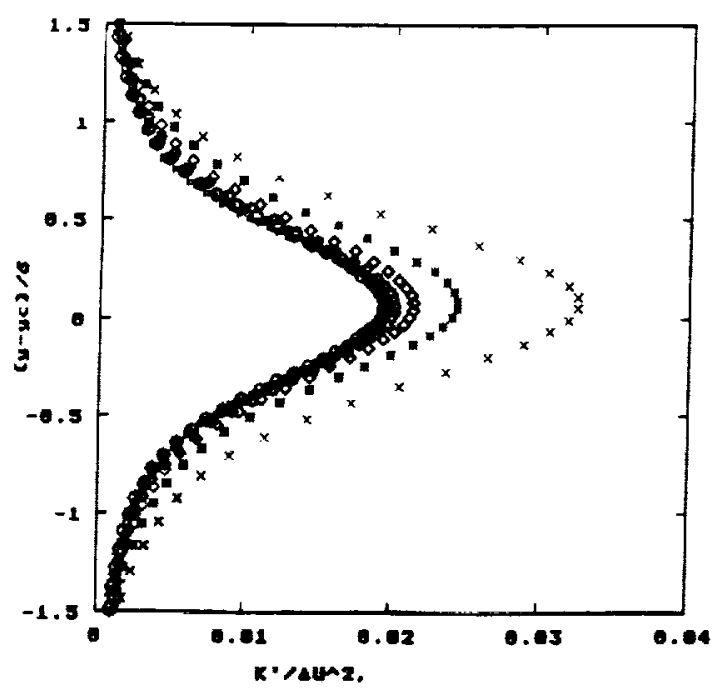

Fig. 16: Coalesced turbulent kinetic energy profiles of different axial locations.

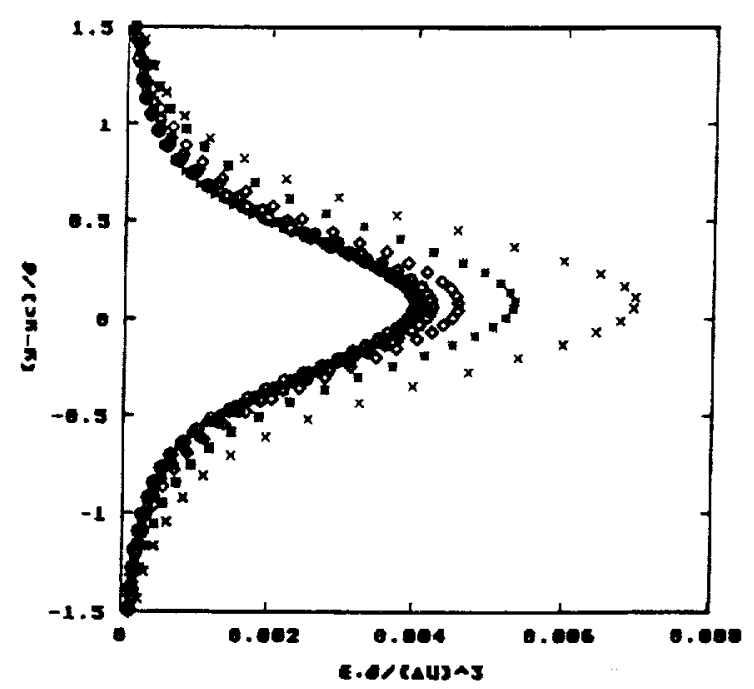

Fig. 17: Coalesced turbulence dissipation profiles of different axial locations.

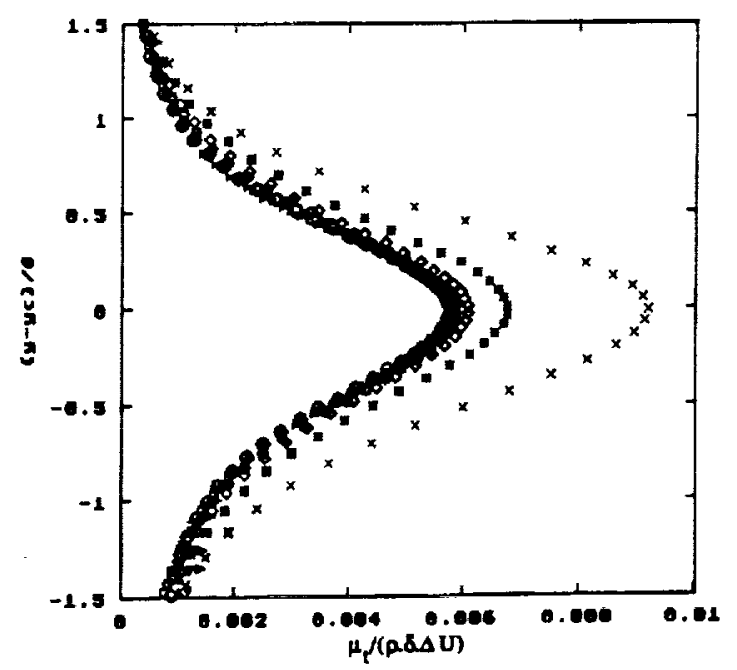

Fig. 18: Coalesced eddy viscosity profiles of different axial locations.

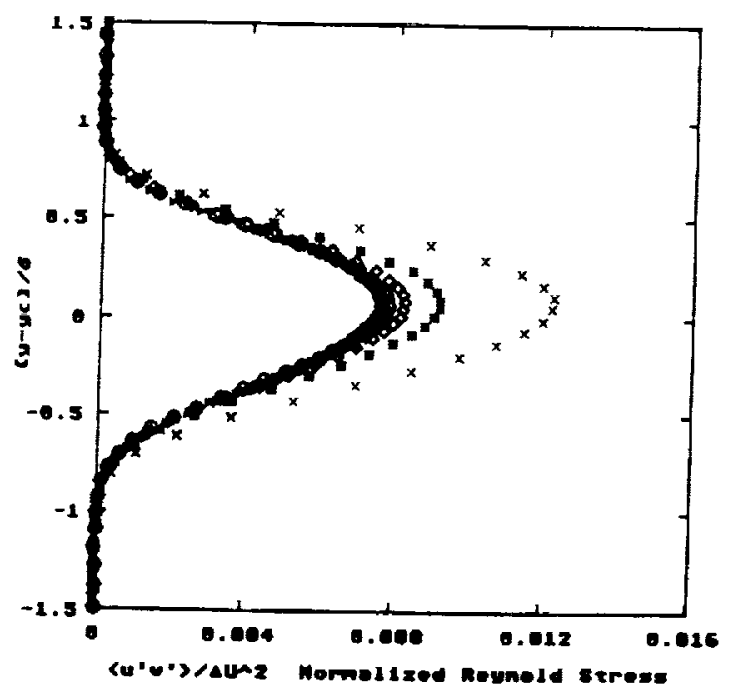

Fig. 19: Coalesced Reynolds stress profiles of different axial locations.

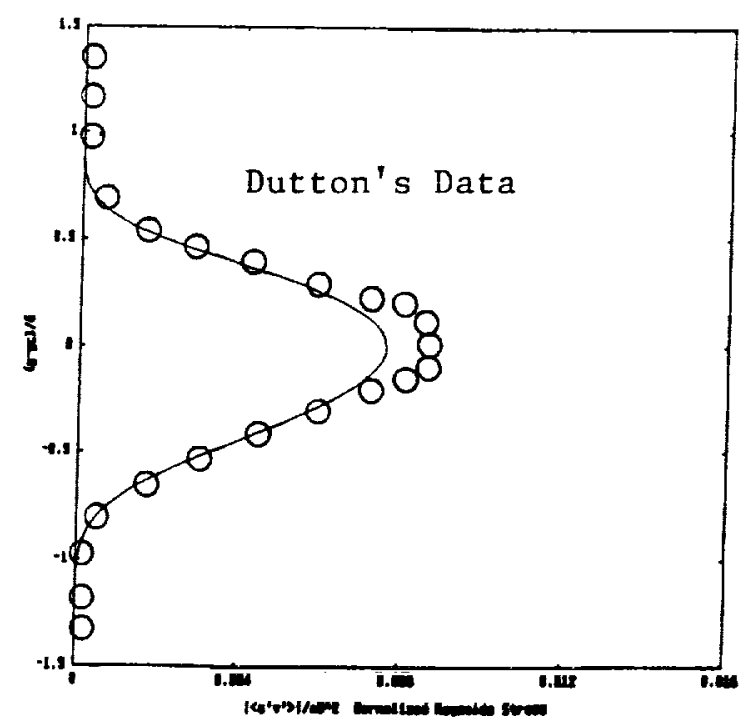

Fig. 20: Comparison of Reynolds stresses between experimental data and predicted results. 


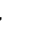

$$
\text { , }
$$
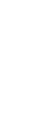


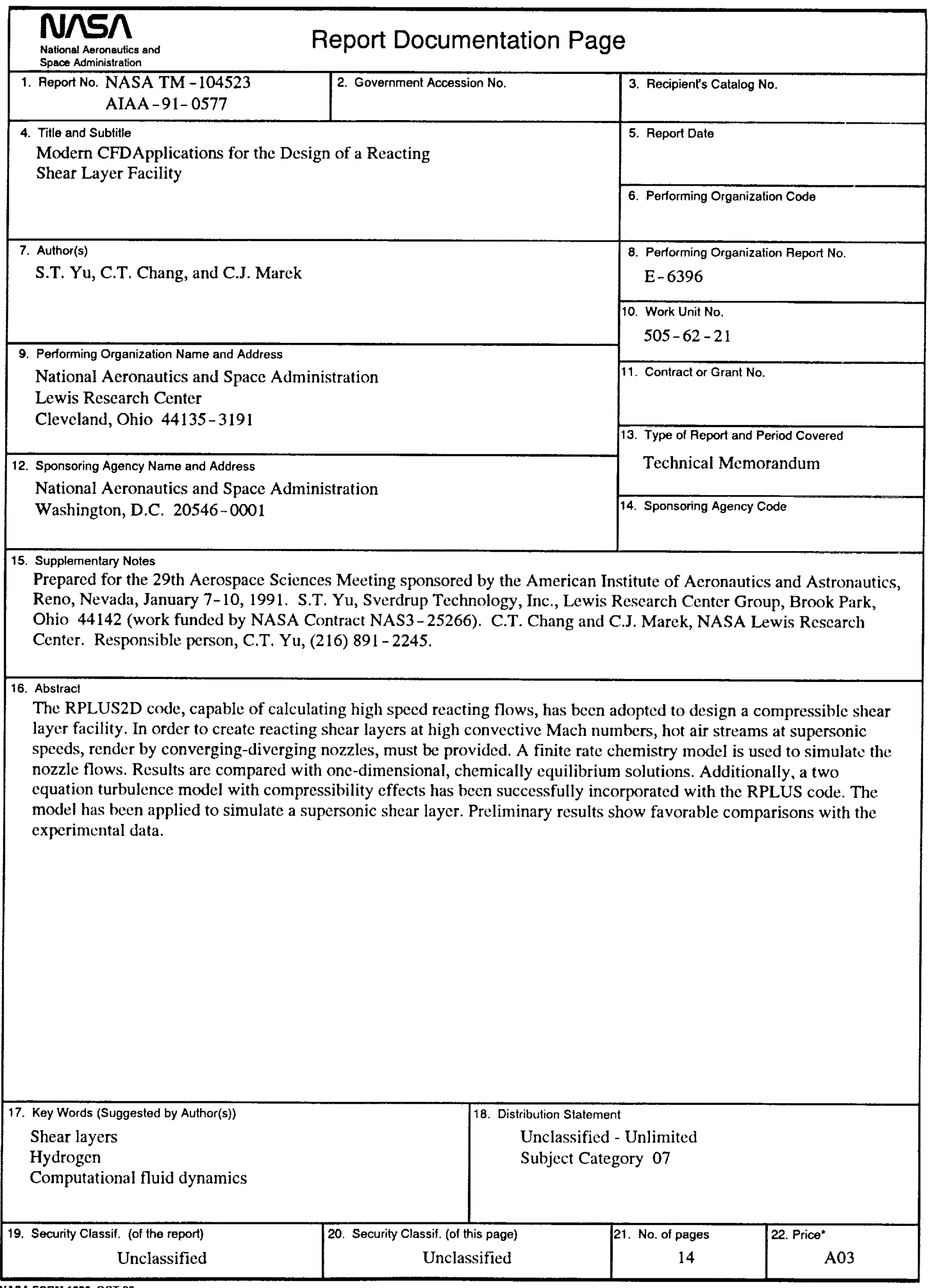

